\title{
Was ist Rechtspopulismus? Einleitung der Redaktion zum PVS-Forum
}

\author{
Samuel Salzborn
}

Online publiziert: 16. Mai 2018

(C) Deutsche Vereinigung für Politikwissenschaft 2018

In der politikwissenschaftlichen Forschung werden, wenn auch in unterschiedlichem Umfang, in fast allen Subdisziplinen Teilaspekte von zwei Phänomenen diskutiert: einerseits die parlamentarische und gesellschaftliche Rechtsradikalisierung und andererseits die Zunahme von populistischer Rhetorik und entsprechenden Politikangeboten in demokratischen Regimen. Nicht immer treten beide Phänomene gemeinsam auf, oft werden sie in den Fachdebatten auch unabhängig voneinander diskutiert, denn nicht jede populistische Strategie entstammt der extremen Rechten und nicht jede rechtsextreme Partei oder Bewegung tritt populistisch in Erscheinung. Gleichwohl ist zu konstatieren, dass es gerade im europäischen und amerikanischen Kontext vielfältige Phänomene gibt, bei denen rechte Politikfelder wie Rassismus, Antisemitismus oder Antifeminismus mit einer populistischen Rhetorik korrelieren - und zwar sowohl im parlamentarischen wie außerparlamentarischen Raum, sowohl bei Akteurinnen und Akteuren mit oder ohne Regierungsverantwortung. Und dabei muss man den vergleichenden Blick nicht erst auf die USA, Donald Trump und sein Umfeld richten, denn in fast allen europäischen Staaten sind mittlerweile rechtsextreme Parteien in den nationalen Parlamenten vertreten, teilweise auch mit Regierungsbeteiligung.

Im deutschsprachigen Kontext liegen bisher viele Detailstudien zu Phänomenen an den Schnittstellen von Rechtsradikalisierung und Populismus vor, die oft unter dem Sammelbegriff des „Rechtspopulismus“ gefasst werden. Gleichwohl fehlt es an einer systematisierenden Zusammenführung der bisherigen Debatten über das Forschungsfeld „Rechtspopulismus“ in methodologischer Absicht, was insbesondere auffällt, da der jeweilige Forschungsansatz in einem fraglos nicht nur wissenschaftlich kontroversen, sondern auch politisch umstrittenen Feld wesentlichen Einfluss

S. Salzborn $(\bowtie)$

Berlin, Deutschland

E-Mail: salzborn@tu-berlin.de 
auf die Forschungsergebnisse nimmt - und damit das jeweilige Verständnis dessen prägt, was Rechtpopulismus genannt wird.

Aus diesem Grund hat die Politische Vierteljahresschrift (PVS) sich entschieden, ein Forum zum Thema „Was ist Rechtspopulismus?“ mit dem Ziel zu organisieren, die weitere Systematisierung des Forschungsfeldes vor allem mit Blick auf grundlegende Fragen anzuregen und die inhaltliche Fachdebatte auch und gerade um ihre methodologischen und selbstreflexiven Dimensionen zu erweitern. Die zahlreichen Forschungsaufsätze, die zur Rechtspopulismusforschung in jüngster Vergangenheit erschienen sind, zeigen nicht nur die fachliche Relevanz und politische Brisanz des Themas, sie bieten auch den Anlass für das PVS-Forum, fachliche Kontroversen durch ihre Bündelung sichtbarer zu machen, als dies möglich wäre, wenn die unterschiedlichen Ansätze an verschiedenen Publikationsorten und damit unvermittelt nebeneinander stünden.

Für das Forum „Was ist Rechtspopulismus?“ wurden vier Fachkolleginnen und -kollegen, die seit Jahrzehnten im Forschungsfeld arbeiten, gebeten, auf der Basis von Schlüsselfragen, die sich im Feld der konzeptionellen und empirischen Rechtspopulismusforschung ergeben, pointiert Stellung zu beziehen - und es wurde ihnen dabei die Freiheit gelassen, einzelne Aspekte stärker, andere weniger stark und manche auch gar nicht zu problematisieren. Denn ein Grundgedanke von methodologischen Debatten kann und sollte ja sein, die jeweils spezifischen Erkenntnisdimensionen auch dadurch in die Diskussion einzubringen, dass man sich nicht zwingend auf jede Vorgabe einlässt und insofern selbst Priorisierungen vornimmt. Als Leitfragen der bisherigen Rechtspopulismusdebatte, die den Autorinnen und Autoren im Vorfeld als Anregung kommuniziert wurden, standen die nach dem Verhältnis von Rechtsextremismus und Rechtspopulismus sowie die nach Gemeinsamkeiten und Differenzen im vergleichenden Blick auf rechtspopulistische Parteien und Gruppen. Mit Blick auf die weltanschauliche Dimension wurde angeregt, nach der Relevanz von Antisemitismus und/oder Islamfeindlichkeit im Rechtspopulismus zu fragen und auf die Bedeutung von Geschlecht zu fokussieren, sowohl mit Blick auf das Personal rechtspopulistischer Parteien/Bewegungen als auch mit Blick auf deren AgendaSetting. Schließlich lag es nahe, die Rolle der Medien für den Rechtspopulismus als bedenkenswert anzuregen, wie auch die nach Strategien, die für den Umgang mit Rechtspopulismus denkbar sind.

Die Kolleginnen und Kollegen, die am Forum beteiligt sind, forschen bereits seit Jahrzehnten zum Gegenstand des Rechtsextremismus bzw. Rechtsradikalismus schon lange bevor das Schlagwort des „Populismus“ aufkam, das mitunter gerade in medialen Debatten auch eine Verharmlosung rechter Politikinhalte nach sich zieht, wenn der Populismusbegriff nicht als strategischer, sondern als rein konzeptioneller (Abgrenzungs-)Begriff verstanden wird. Während der Forschungsschwerpunkt von Ruth Wodak in der Untersuchung von öffentlichen (politischen und medialen) Diskursen liegt und sie sich bereits in den 1980er-Jahren mit Antisemitismus und Sexismus sowie in den 1990er-Jahren intensiv mit der Freiheitlichen Partei Österreichs (FPÖ) befasst hat, arbeitet Michael Minkenberg ebenfalls seit den 1980erJahren zur vergleichenden Rechtsextremismus- bzw. Rechtsradikalismusforschung, zunächst mit intensivem Fokus auf Deutschland, Frankreich und die USA, später auch mit erweitertem Blick auf Nord- und Osteuropa. Frank Decker fokussiert in 
der Rechtsextremismus- bzw. Rechtspopulismusforschung stark auf die parlamentarische Dimension und die Parteienforschung, auch mit europäisch-vergleichendem Blick sowie mit besonderem Schwerpunkt auf die jeweiligen Parteien- und Parlamentarismuskontexte und damit die Relationen zu politischen Systemfragen. Ursula Birsl war in den 1990er-Jahren eine der ersten, die die Geschlechterperspektive für die Rechtsextremismusforschung systematisch untersucht hat und seitdem immer wieder, auf der Basis eigener empirischer Studien mit besonderem Bezug zur vergleichenden Migrations- und Geschlechterforschung, die Notwendigkeit der Selbstreflexion im Forschungsfeld einfordert.

Alle vier Kolleginnen und Kollegen wussten bei der Bitte der PVS, für das geplante Forum als Autorin bzw. Autor tätig zu werden, nicht, wer noch zur Beteiligung eingeladen worden war, um damit ein höchst mögliches Maß an erkenntnistheoretischer, methodologischer und konzeptioneller Freiheit zu schaffen, die gerade auch Differenzen in den jeweiligen Perspektiven fruchtbar für die weitere Forschung werden lassen kann. 